\title{
Effects of Glucosamine and Celadrin on Platelet Function
}

\author{
Pei-Chun Lin, Samuel O Jones, David L McGlasson
}

\author{
OBJECTIVE: The purpose of this study was to \\ determine the effects of glucosamine and celadrin on \\ platelet function.
}

DESIGN: Baseline values were determined on the Chronolog 570VS platelet aggregometer with whole blood aggregation impedance readings using 2 different concentrations of ADP $(5 \mu \mathrm{M}, 10 \mu \mathrm{M})$, collagen $(1 \mu \mathrm{g} / \mathrm{mL})$, arachidonic acid $(0.5 \mathrm{mM} / \mathrm{L})$ and an Accumetrics whole blood platelet aggregation cartridge assay for P2Y12 receptors were obtained from 24 healthy volunteers. These subjects then took the suggested doses of Glucosamine with Celadrin (Stockbridge Naturals) as advertised (estimated $1500 \mathrm{mg}$ daily) for 2 weeks. Platelet aggregation analyses, as described above, were obtained after treatment. Statistics performed via a McNemar test.

MAIN OUTCOME: Five of twenty-four subjects had at least a $20 \%$ difference in whole blood aggregation using the $5 \mu \mathrm{M}$ concentration of ADP. A total of 6 and 7 subjects also showed a significant difference in platelet aggregation with administration of collagen and arachidonic acid, respectively. No significant differences were found with Accumetrics assay for P2Y12 in any of the subjects.

\footnotetext{
The peer-reviewed Research and Reports Section seeks to publish reports of original research related to the clinical laboratory or one or more subspecialties, as well as information on important clinical laboratory-related topics such as technological, clinical, and experimental advances and innovations. Literature reviews are also included. Direct all inquiries to David L McGlasson MS, MLS, 59th Clinical Research Division/SGRL, 2200Berquist Dr., Bldg. 4430, Lackland AFB TX 78236-9908, david.mcglasson@lackland.af.mil
}

CONCLUSION: Glucosamine and celadrin may inhibit platelet aggregation in some individuals via aspirin-like effects as well as inhibition of ADP receptor P2Y1 but not P2Y12.

ABBREVIATIONS: ADP = adenosine diphosphate, NSAID = non-steroidal anti-inflammatory drug, EDTA = ethylenediaminetetraacetic acid, WBA = whole blood aggregation, $\mathrm{CBC}=$ complete blood count, $\mathrm{PGE}=$ prostaglandin $-\mathrm{E}$

INDEX TERMS: Blood platelets, Platelet aggregation, Glucosamine, Celadrin

Clin Lab Sci 2010;23(1):32

Pei-Chun Lin, M.D., Wilford Hall Medical Center, Lackland AFB, TX.

Samuel O. Jones, M.D., Wilford Hall Medical Center, Lackland AFB, TX.

David L. McGlasson, M.S. CLS/NCA, Department of Air Force, Lackland AFB, TX.

Address for Correspondence: Pei-Chun Lin, M.D., Wilford Hall Medical Center, Cardiology Department, 2200 Bergquist Drive, Ste 1, Lackland AFB, TX 78236,Email:pei-chun.lin@lackland.af.mil

\section{INTRODUCTION}

The use of dietary supplements in the United States is on the rise. A national survey conducted by the Center for Disease Control in $2007^{1}$ found that $17.7 \%$ of American adults had used "natural products" in the previous 12 months as compared to $2.1 \%$ in $1990 .^{2}$ The most popular products used were fish oil/omega 3/DHA (37.4\%), glucosamine (19.9\%), and echinacea (19.8\%). ${ }^{1}$ Herbal and dietary 
supplements are not regulated by the Food and Drug Administration, thus raising concerns over potential side effects and drug interactions with conventional medicines. This use of supplements by adults suggested to our cohort that glucosamine and celadrin may affect platelet function.

One of our subject controls, who has donated blood as a normal platelet aggregometry control for years, suddenly had abnormal values suggesting platelet inhibition. Whole blood aggregation using $5 \mu \mathrm{M}$ and $10 \mu \mathrm{M}$ concentrations of ADP showed $5 \Omega$ and $0 \Omega$, respectively ( normal aggregation is $\geq 8 \Omega$ ). Upon further questioning, patient stated that she had started taking Glucosamine with Celadrin supplement for osteoarthritis. After cessation of the supplement, her platelet function returned to normal.

Glucosamine is an amino-sugar that is produced naturally in humans and is a common supplement used for treatment of osteoarthritis. Celadrin, a proprietary blend of esterified fatty acids, is often used in combination with glucosamine. The same population developing osteoarthritis is at also risk for cardiovascular diseases. Therefore, the likelihood for use of anti-coagulation or anti-platelet agents exists. Little is known about the effects of glucosamine on platelet aggregation in humans, ${ }^{3-6}$ and there is no literature of the effects of celadrin on platelet function reported. Given the potential drug interactions, the implication that glucosamine and celadrin may have effects on platelet function is an important one, especially in those who take concomitant anticoagulation and/or anti-platelet agents, such as aspirin, clopidogrel, and newer agents like prasugrel and ticagrelor. This study is designed to evaluate only the effects of Glucosamine with Celadrin on platelet function.

\section{MATERIALS AND METHODS}

The presented protocol was approved through the local institutional review board in accord with the tenets of the Helsinki protocol for human subject experimentation. Informed consent obtained from all subjects. This study was monitored and approved by the United States Air Force Surgeon General's Office. All authored materials constitute the personal statements of Pei-Chun Lin and are not intended to constitute an endorsement by the U.S. Air Force or any other Federal Government entity.

\section{Participants}

Inclusion criteria for the volunteers included age over 18 years, no contraindication to taking Glucosamine with Celadrin, not currently pregnant or breast feeding, not currently taking (or requiring from a medical standpoint) any anti-platelet regimen or anticoagulant, not taking any NSAID or aspirin for 7 days prior to the test, hematocrit > 33\%), and platelets $>100,000$. Twenty-seven normal healthy volunteers were asked to donate a blood sample consisting of approximately $15 \mathrm{~mL}$ drawn into 2 blue top tubes containing $3.2 \%$ sodium citrate, one 2.0 $\mathrm{mL}$ partial fill citrated tube (describe the citrate conc.), and 1 lavender top tube containing (describe $\%$, sodium or potassium, etc.) EDTA. Laboratory tests performed included whole blood platelet aggregation with 2 different concentrations of ADP $(5 \mu \mathrm{M}, 10 \mu \mathrm{M})$, collagen $(1 \mu \mathrm{g} / \mathrm{mL})$, arachidonic acid $(0.5 \mathrm{mM} / \mathrm{L})$ and Accumetrics whole blood platelet aggregation assay specific for P2Y12 receptors. After the baseline blood draw collection, the subjects were given Glucosamine with Celadrin (Stockbridge Naturals) supplement of a total daily dose of $1500 \mathrm{mg}$ for 14 days total. At the end of the 14 days, the blood tests were repeated. Concentration of glucosamine in the supplement was also analyzed in our CRD (Clinical Research Division) laboratory to ensure consistent amount of glucosamine taken by each patient. Pill counts were done before and after 14 days to measure level of compliance. Subjects were excluded from the study if more than $10 \%$ of the pills were not taken.

\section{Whole-blood assays}

Whole-blood impedance platelet aggregometry was performed using a WBA whole-blood impedance platelet aggregometer (Chrono-Log Corporation, Havertown, Pennsylvania, USA). Whole-blood citrated samples were diluted 1:1 with normal saline for 
total volume of $1000 \mu \mathrm{l}$ in each aggregometry cuvette with added stir bar. Next, $10 \mu \mathrm{l}$ of an agonist was added to the cuvette and impedance was recorded for 6 min. $^{7}$ Agonists used were ADP in 2 different concentrations $(5 \mu \mathrm{M}$ and $10 \mu \mathrm{M})$, collagen $(1 \mu \mathrm{g} / \mathrm{mL})$, and arachidonic acid $(0.5 \mathrm{mM} / \mathrm{L})$. For each agonist, normal aggregation was defined as $\geq 8 \Omega$ (ohms) impedance. $^{8}$

Accumetrics whole blood platelet aggregation assay for P2Y12 was performed using the VerifyNow Assay. This is designed to measure the level of P2Y12 receptor blockade. The VerifyNow instrument is a turbidmetric based optical detection system that measures platelet-induced aggregation by an increase in light transmittance as platelet aggregate. ${ }^{9}$ The selfcontained sealed assay device employs microbead agglutination technology and contains a lyophilized preparation of human fibrinogen-coated beads, platelet activators (adenosine-5-diphosphate ADP/ PGE1) and buffer. The patient specimen is aliquoted into Greiner Bio-One Vacuette partial fill blood collection tubes ( $2 \mathrm{~mL}$ fill volume) containing 3.2\% sodium citrate that is automatically dispensed and incorporated with the platelet activator reagent. The light transmittance increases as activated platelets aggregate and bind to fibrinogen-coated beads. The instrument then measures the baseline platelet function for each sample and reports the percent inhibition result. ${ }^{10}$

\section{Statistics and data analysis}

SPSS Sample Power (version 2.0) was employed to estimate the samples sizes for detecting a treatment effect with glucosamine using two tests of platelet function. The criterion for significance (alpha) was set at $\leq 0.050$ and power $>0.80$. A McNemar test was applied to assess binary results of whole blood aggregation and Accumetrics platelet function assay. Platelet aggregation results used an impedance value of $8 \Omega$ (ohms) as criterion for normal limits. The Accumetrics P2Y12 cartridge normal limits were characterized as less than $20 \%$ of platelets affected (How/where was this determined?). A sample size of 25 paired observations was needed to yield a significant difference assuming that the treatment difference of platelet function is $20 \%$ in the sample population.

\section{RESULTS}

A total of 27 subjects were recruited over a period of one month, and all had baseline blood samples performed. Ages ranged from 22 to 56, median age of 35. Thirty-two percent were men and $68 \%$ were women. All had normal baseline CBC values. Of the included subjects, two had baseline whole-blood impedance platelet aggregation of less than $8 \Omega$ utilizing the $5 \mu \mathrm{M}$ concentration of ADP; one of these two also had $<8 \Omega$ with collagen and arachidonic acid agonists. All 27 subjects had normal baseline Accumetrics P2Y12 values with less than 20\% platelet inhibition.

All lab values were retested in those who completed the two-week course of the Glucosamine with Celadrin. Three of the female subjects withdrew from the study prior to taking Glucosamine with Celadrin (two due to other medical reasons, one unknown). Of the 24 subjects who completed the study, a total of five $(20.8 \%)$ and six (25\%) subjects showed significant difference with the $5 \mu \mathrm{M}$ and $10 \mu \mathrm{M}$ concentrations of ADP, respectively. Two out of the five subjects who showed a significant difference with the $5 \mu \mathrm{M}$ concentrations of ADP also showed a difference in platelet aggregation with administration of collagen and arachidonic acid. A total number of 6 (25\%) and 7 (29\%) subjects had decreased platelet aggregation post-treatment in response to collagen and arachidonic acid agonists, respectively. No significant differences were found with pre- and posttreatment $\mathrm{CBC}$ or Accumetrics assay for P2Y12 values in all the subjects (Table 1). Compliance was met in all 24 subjects.

\section{DISCUSSION}

Effects on platelets by various agents have been an interest in the recent past due to the potential for significant clinical implications, such as prevention of ischemic events or alteration of bleeding complications. Platelets are circulating anucleate disc- 
Table 1. Response to agonists after taking Glucosamine with Celadrin

\begin{tabular}{|c|c|c|c|c|c|}
\hline \multirow[b]{3}{*}{$\begin{array}{c}\geq 20 \% \text { decline from } \\
\text { baseline (\% of } \\
\text { patients) }\end{array}$} & \multicolumn{5}{|c|}{ Chronolog whole-blood aggregometry } \\
\hline & $5 \mu \mathrm{M}$ ADP & $10 \mu \mathrm{M} A D P$ & $1 \mu \mathrm{g} / \mathrm{mL}$ Collagen & $0.5 \mathrm{mM} / \mathrm{L}$ Arachidonic acid & Accumetric VerifyNow \\
\hline & 20.8 & 25 & 25 & 29 & 0 \\
\hline
\end{tabular}

shaped cells that are responsible for the initiation of primary hemostasis, which repair injury to the vascular endothelium. When there is disruption to the integrity of the endovascular lining, platelets are exposed to the underlying collagen fibrils. ${ }^{11}$ These collagen fibrils not only provide a surface for platelet adhesion, but also serve as a strong stimulus for platelet activation, which includes secretion of thromboxane $\mathrm{A} 2$ and ADP into the circulation. ${ }^{12}$ The released thromboxane $\mathrm{A} 2$ and $\mathrm{ADP}$ stimulate neighboring platelets, causing them to become activated and secrete additional thromboxane $\mathrm{A} 2$ and ADP. ${ }^{13}$ ADP interacts with two G-protein-coupled platelet purinergic receptors, $\mathrm{P} 2 \mathrm{Y} 1$ and $\mathrm{P} 2 \mathrm{Y} 12 .{ }^{14}$ When ADP stimulates the platelet $G$ alpha-subclass q-coupled P2Y1 receptor, it causes protein phosphorylation, phosphoinositide hydrolysis, thromboxane A2 formation, and an increase in cytosolic $\mathrm{Ca}^{++}$. Likewise, ADP stimulates the $\mathrm{G}$ alpha-subclass i-coupled P2Y12 receptor that inhibits cAMP formation. Simultaneous activation of both P2Y1 and $\mathrm{P} 2 \mathrm{Y} 12$ is required for the full ADP-response in platelets as seen in studies using platelets derived from knockout mice. ${ }^{14}$

In this study, we proposed that glucosamine and celadrin, commonly used supplements for osteoarthritis, have inhibitory effects on platelets and can exacerbate effects of anti-platelet agents. We found that 6 out of the 24 subjects demonstrated at least a $20 \%$ decline in whole blood aggregation using the $10 \mu \mathrm{M}$ concentration of ADP and 5 out of the 24 subjects also showed the same response with the $5 \mu \mathrm{M}$ concentration of ADP post-treatment with Glucosamine with Celadrin. Several subjects also showed decreased platelet aggregation with the agonists, collagen and arachidonic acid (a total of 6 and 7 subjects, respectively). However, no response was found with the Accumetrics whole blood platelet aggregation VerifyNow Assay for P2Y12. This demonstrated that in some individuals Glucosamine with Celadrin will decrease platelet response to collagen and arachidonic acid (mimicking aspirin-like effects) as well as affecting ADP P2Y1 receptors, but not P2Y12 receptors.

Previous studies support our findings. Lu-Suguro et al utilized guinea pigs and found glucosamine decreased platelet aggregation in response to ADP by $51 \%$ and this study also prevented ADP-induced extracellular release of ATP and thromboxane A2 production by $91 \%$ and $96 \%$, respectively. ${ }^{3}$ Another study done in rabbits by Kinlough-Rathbone et al also found decreased platelet aggregation induced by ADP with glucosamine administration. ${ }^{4} \mathrm{McNamara}$ et al investigated the hemostatic effects of oral chrondroprotective agents in dogs for 30 days. There were significant reductions of platelet aggregation in response to $\mathrm{ADP}$ and collagen as well as overall decline in total ATP release and platelet count. ${ }^{5}$ Hua et al took human platelet-rich plasma and stimulated it with ADP in the presence of glucosamine. They found that glucosamine suppressed platelet aggregation in response to $\mathrm{ADP}$ by inhibiting binding of its receptors. It also inhibited the extracellular release of granule contents (ATP and platelet factor 4) and production of thromboxane A2 from ADPstimulated platelets. Moreover, glucosamine halted the intracellular calcium mobilization by blocking phosphorylation of Syk (tyrosine kinase in platelets) upon ADP-stimulation. ${ }^{6}$ Literature search via Medline from 1950 to current found no studies on celadrin and its effects on platelet function. 
Our study is the first study, to our knowledge, showing Glucosamine with Celadrin may exhibit inhibitory effects on platelet aggregation in humans via possible aspirin-like effects and inhibition of ADP receptor P2Y1 but not P2Y12. This is an important finding in that many of those who take glucosamine and/or celadrin may also be those who take antiplatelet and/or anti-coagulation agents. The clinical implication of this cohort may be larger than we think, especially in the era of newer and more potent anti-platelet agents, such as prasugrel and tecagrelor. It is uncertain why some individuals in our study had platelet inhibition and others did not. This may be due to gender, age, race differences of the individuals as well as possible genetic predisposition not yet elucidated. This may also have been due to the addition of celadrin to glucosamine, which has not been previously studied in this manner. The fact that our sample size is small also precludes us from drawing definitive conclusions of susceptible individuals. Further studies are needed, with larger sample size, randomized-control design, and inclusion for clinical outcomes will better delineate the importance of this subject.

Clin Lab Sci encourages readers to respond with thoughts, questions, or comments regarding this article. Email responses to westminsterpublishers@comcast.net. In the subject line, please type "CLIN LAB SCI 23(1) RE LIN". Selected responses will appear in the Dialogue and Discussion section in a future issue. Responses may be edited for length and clarity. We look forward to hearing from you.

\section{REFERENCES}

1. Barnes PM, Bloom B, Nahin R. Complementary and alternative medicine use among adults and children: United
States 2007. CDC National Health Statistics Report \#12, 2008.

2. Eisenberg DM, Davis RB, Ettner SI, et al. Trends in alternative medicine use in the United States, 1990-1997: results of a followup national survey. JAMA 1998; 180:1569-75.

3. Lu-Suguro JF, Hua J, Sakamoto K, Nagaoka I. Inhibitory action of glucosamine in platelet activation in guinea pigs. Inflamm Res 2005;54(12):493-9.

4. Kinlough-Rathbone RL et al. Effect of amio sugars on platelet aggregation and on fibrinogen binding. Thromb Hemost 1984;52(1):75-80.

5. McNamara PS et al. Hematologic, hemostatic, and biochemical effects in dogs receiving an oral chondroprotective agent for thirty days. Am J Vet Res 1996;57(9):1390

6. Hua J, Suguro S, Iwabuchi K, Tsutsumi-Ishii Y, Sakamoto K, Nagaoka I. Inhibitory actions of glucosamine on platelet functions. Inflamm Res 2004;53(12):680-8.

7. Ivandic BT, Giannitsis E, Schlick P, Staritz P, Katus HA, Hohlfeld T. Determination of aspirin responsiveness by use of whole blood platelet aggregometry. Clin Chem 2007; 53:614-9.

8. McGlasson DL, Fritsma GA. Whole Blood Platelet Aggregometry and Platelet Function Testing. Semin Thromb Hemosts 2009;35(2)168-80.

9. Michelson AD, Frelinger AL, Furman MI. Current options in platelet function testing. Am J Cardiol 2006;98:4N-10N.

10. McGlassonDL, Fritsma GA. Comparison of four laboratory methods to assess aspirin sensitivity. Blood Coagul Fibrinolysis 2008;19:120-3.

11. Jung SM and Masaaki M. Recent advances in thrombosis and hemostasis: platelet collagen receptors. 2008.

12. Garcia A, Haripriya S, Swaminathan M, Soochong K, Satya PK. Regulation and functional consequences of ADP receptor-mediated ERK2 activation in platelets. Biochem $J$ 2007;404(2):299-308.

13. Jackson SP. The growing complexity of platelet aggregation. Blood 2007;109(12):5087-95.

14. Kunapuli SP, Dorsam RT, Kim S, Quinton TM. Platelet purinergic receptors. Curr Opin Pharmacol 2003;3(2):17580 . 